ORIGINAL ARTICLE

\title{
Increasing risk behaviour and high levels of undiagnosed HIV infection in a community sample of homosexual men
}

\author{
J P Dodds, D E Mercey, J V Parry, A M Johnson
}

Sex Transm Infect 2004;80:236-240. doi: 10.1136/sti.2003.007286

See end of article for authors' affiliations

....................

Correspondence to: Julie Dodds, Centre for Sexual Health and HIV Research, Department of Primary Care and Population Sciences, Royal Free and University College Medical School, Mortimer Market Centre, Mortimer Market, off Capper Street, London WCIE 6AU, UK; jdodds@gum.ucl.ac.uk

Accepted for publication 6 November 2003

\begin{abstract}
Objectives: To estimate changes in sexual behaviour over time. To examine the proportion of undiagnosed HIV infection in a community sample of homosexual men. To explore the relation between HIV status, diagnosis, and sexual behaviour.

Methods: Five cross sectional surveys of men attending selected gay community venues in London between 1996 and 2000 ( $n=8052)$. Men were recruited in 45 to 58 social venues (including bars, clubs, and saunas) across London. Participants self completed an anonymous behavioural questionnaire. In 2000, participants in community venues provided anonymous saliva samples for testing for anti-HIV antibody. Results: The proportion of men having unprotected anal intercourse (UAl) increased significantly each year from $30 \%$ in 1996 to $42 \%$ in 2000 ( $p<<0.001$ ). In 2000, 132 of 1206 (10.9\%) saliva samples were HIV antibody positive. Of the HIV saliva antibody positive samples, 43/132 (32.5\%) were undiagnosed. Around half of both diagnosed and undiagnosed HIV saliva positive men reported UAl in the past year. Of the $83 \%$ of men who reported their current perceived HIV status, $4.1 \%$ reported an incorrect status. HIV antibody positivity was associated with increasing numbers of UAI partners, and having a sexually transmitted infection (STI) in the past year (OR 2.15).

Conclusions: Homosexual men continue to report increasing levels of UAI. HIV prevalence is high in this group, with many infections remaining undiagnosed. The high level of risky behaviour in HIV positive men, regardless of whether they are diagnosed, is of public health concern, in an era when HIV prevalence, antiretroviral resistance, and STI incidence are increasing.
\end{abstract}

n 2000, 43\% of diagnosed prevalent infections in the United Kingdom were in men who have sex with men. ${ }^{1}$ Recent estimates suggest that HIV incidence among homosexual men remains at a high level of $2 \%^{2}$ per annum and there is evidence of increasing levels of resistant virus in primary infections. ${ }^{3}$ Reported recent increases in sexually transmitted infections, ${ }^{4}$ HIV prevalence, ${ }^{5}$ and high risk sexual behaviour $^{6}$ are all causes for concern, and highlight the potential for continuing spread of the virus.

In the era of highly active antiretroviral therapy (HAART), people are living longer with HIV/AIDS, and it has become increasingly important to understand the relation between current HIV status and risky sexual behaviour. Researchers and practitioners have described strategies ${ }^{7-9}$ used by homosexual men to reduce the risk of HIV transmission during UAI, for example "negotiated safety,"10 which relies on men testing for HIV and engaging in UAI only with partners of the same HIV status as themselves. Therefore, it is important to assess the accuracy of self reported HIV status and the level of undiagnosed infection to determine the effectiveness of these strategies.

Our understanding of HIV prevalence in homosexual men in the United Kingdom relies almost entirely on clinic population samples. ${ }^{5}$ These surveillance initiatives give little indication of risk behaviour among those currently living with HIV and may not be representative of the broader gay community who do not use clinics. Behavioural surveillance data clearly demonstrate increased risk behaviour in gay community samples in recent years, ${ }^{6}$ but there are no recent data to link behaviour with HIV status in community samples. ${ }^{11}$

In this paper we report on the changes in sexual behaviour over time; examine the prevalence of diagnosed and undiagnosed HIV among a non-clinic sample of homosexual men; explore the relation between HIV status, HIV diagnosis, and key sexual behaviours; and assess the accuracy of self reported HIV antibody status.

\section{METHODS}

Details of the survey methodology have been published elsewhere. ${ }^{12}$ Briefly, we undertook an annual behavioural survey of homosexual men (from 1996 to 2000) who socialised in gay venues across London. Venues were chosen to represent a cross section of different types of venue (that is, bar, club, and sauna). Wherever possible we surveyed the same venues each year to maximise comparability between surveys. Our sampling frame also included genitourinary medicine (GUM) clinics, but these are excluded from the analyses presented here. Trained fieldworkers visited selected venues between November and March annually. All men in the venue or queuing to enter the venue, or all men in a specific area in the larger venues were invited to participate and all refusals recorded.

Participants filled in a short anonymous self completion questionnaire covering demographic details, sexual health service use, HIV testing history, self perceived HIV status, and sexual behaviour.

In 2000 we introduced unlinked anonymous saliva testing for HIV antibody. Men were offered a kit consisting of the questionnaire, an OraSure oral fluid collection device (Orasure Technologies Inc, Bethlehem, PA, USA) ${ }^{13}$ with instructions and an information sheet. Saliva specimens were linked to the questionnaire via a barcode with no personal identifiers. Participants were given details of where they could go for a named HIV test should they wish to.

Three measures were used to assess the prevalence of high risk sexual behaviour: the number of men who had engaged in unprotected anal intercourse (UAI) in the past year with both once only partners (casual) or more frequent partners (regular) and the numbers of such partners; UAI with 
partners of the same, discordant or unknown HIV status; and reporting a sexually transmitted infection in the past year.

\section{Laboratory testing}

All oral fluid samples were sent to the Central Public Health Laboratory for anti-HIV antibody testing by GACELISA HIV 1 and 2 (Abbott Laboratories, Maidenhead, UK). ${ }^{14}$ All samples were also tested for total immunoglobulin G (IgG) as a specimen quality check. Specimens reactive in the GACELISA assay were also examined by a western blot test (Genelabs HIVblot 2.2).

\section{Statistical analysis}

Questionnaire data were double entered using Epi-Info $6^{16}$ and analysed using SPSS software. ${ }^{17}$ Bivariate analyses were undertaken using $\chi^{2}$ tests for categorical variables and Student's $t$ tests or Mann-Whitney $\mathrm{U}$ tests for continuous variables. The multivariate analysis employed was unconditional logistic regression adjusting for factors associated in univariate analysis. The odds ratio (OR) using 95\% confidence intervals (95\% CI) was used to compare estimates of risk behaviours between HIV positive and negative men.

\section{RESULTS}

\section{Trends in sexual behaviour}

Between 45 and 58 community venues were sampled annually. A total of 10776 questionnaires were offered between 1996 and 2000 and 8052 completed questionnaires were returned, giving a response rate of $74.7 \% \quad(75.7 \%$ in $1996,78.3 \%$ in $1997,84.6 \%$ in $1998,73.9 \%$ in 1999 , and $63.3 \%$ in 2000). Similar numbers of questionnaires were returned each year and similar demographics were reported every year. Men were excluded who had already completed the questionnaire in that year (225 respondents) and those who specified their sexual orientation as heterosexual and had not reported sex with another man in the past year (85 respondents).

Since data were published on the 1996 to 1998 surveys $^{6}$ respondents have continued to report increasing levels of UAI (fig 1). Using 1996 as the baseline, there was a significant increase in reporting of UAI after adjusting for age (there were no demographic differences by year of survey, apart from age) $(29.7 \%$ in $1996 ; 33.6 \%$ in 1997 OR 1.22 (95\% CI 1.05 to 1.41$) ; 35.5 \%$ in 1998 OR 1.31 (95\% CI 1.13 to 1.52 ); $37.3 \%$ in 1999 OR 1.44 (95\% CI 1.24 to 1.67 ); $41.8 \%$ in 2000 OR 1.74 (95\% CI 1.50 to 2.01 )) (fig 2). The adjusted odds of having UAI with partners of an unknown or discordant status also increased $(15.8 \%$ in $1996 ; 17.4 \%$ in 1997 OR 1.16 ( $95 \%$ CI 0.96 to 1.39 ); $19.5 \%$ in 1998 OR 1.31 (95\% CI 1.09 to $1.59) ; 19.2 \%$ in 1999 OR 1.31 (95\% CI 1.08 to 1.59$) ; 21.6 \%$ in 2000 OR 1.50 (95\% CI 1.25 to 1.81$)$ ). This increase in UAI

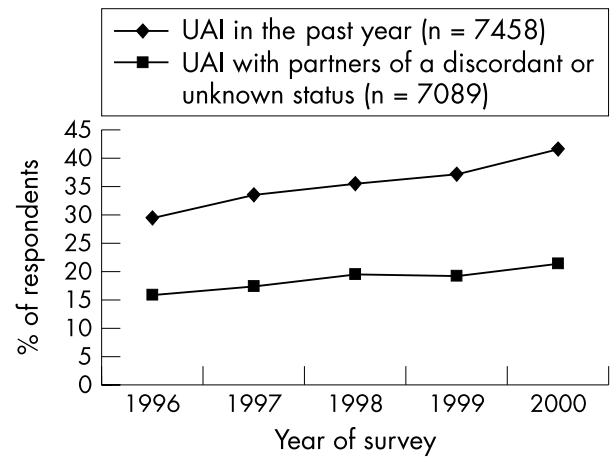

Figure 1 Proportion of men reporting unprotected anal intercourse (UAl) in the past year.

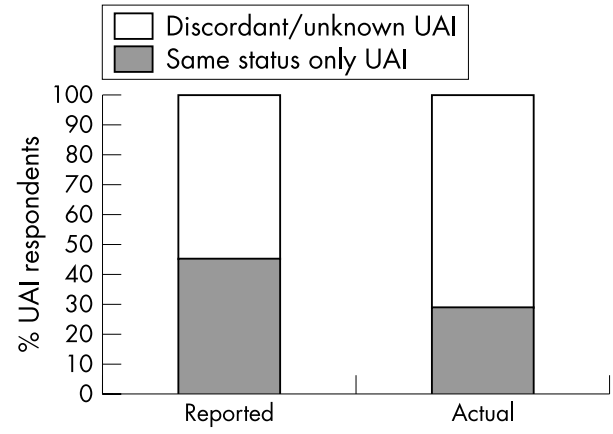

Figure 2 UAl in the past year with partners of the same or discordant/ unknown HIV status reported and actual values.

remained significant when only those venues sampled in all years were included; however, there was no significant increase in UAI with partners of an unknown or discordant HIV status.

\section{Saliva testing}

In 2000 we surveyed 45 community venues, 38 bars, six clubs, and one sauna. Questionnaires and OraSures were offered to 2290 men and 1449 questionnaires were returned, giving an overall response rate of $63 \%$. A saliva sample was returned by 1294 (57\%). A total of 1206 respondents were included for further analysis. Men were excluded who had already completed the questionnaire in that year $(32$ respondents), heterosexual men (11 respondents), saliva specimen not linked to a questionnaire (44 respondents), and saliva specimens with inadequate IgG levels (13 respondents).

Of the men who completed a questionnaire, 11\% (155 men) refused to provide a saliva specimen. Those who refused the saliva test were less likely to be white (OR 0.42 (95\% CI 0.26 to 0.66)); however, there were no other significant demographic differences. Men who declined the saliva test were less likely to have attended a GUM clinic in the past year (OR 0.51 (95\% CI 0.34 to 0.77$)$ ), less likely to have ever tested for HIV (OR 0.56 (95\% CI 0.38 to 0.81$)$ ), and reported fewer UAI partners in the past year (MannWhitney $=-2.55, \mathrm{p}<0.01)$. All these variables, apart from HIV testing, remained significant when placed in a logistic regression model.

\section{HIV status by demographic and sexual health service use}

Of 1206 men tested for salivary HIV antibody, 132 (10.9\%, 95\% CI 9.2 to 12.7 ) were anti-HIV positive. Table 1 shows the sample's demographic composition and sexual health service use by HIV status. HIV saliva antibody positivity was associated with older age (median age of 35 years for HIV positive men, compared with 32 years for HIV negative men). However, men aged between 35-39 had a higher level of positivity than the 40 plus age group. Men who received 3 or more years education after the age of 16 were less likely to be HIV saliva antibody positive than those who received less formal education (OR 0.46). Also, men who were unemployed were more likely to be HIV saliva antibody positive than employed men (OR 2.26). There were no significant differences in saliva antibody positivity between white and non-white men.

Men who had attended a GUM clinic in the past year were more likely to be positive on salivary testing (18.1\%) (OR $3.83)$ than those who had not attended a clinic (5.4\%). Men who reported never having been tested for HIV were less likely to be HIV positive (OR 0.26). 
Table 1 HIV status by demographic profile, clinic attendance, and HIV testing history

\begin{tabular}{|c|c|c|}
\hline & HIV status & \multirow{2}{*}{$\begin{array}{l}\text { Odds ratio for positivity }(95 \% \mathrm{Cl}) \\
\text { OR unadjusted }\end{array}$} \\
\hline & HIV positive/total (\%) & \\
\hline \multicolumn{3}{|l|}{ Age group (years) } \\
\hline$<25$ & $3 / 192(1.6)$ & $0.13(0.003$ to 0.46$)$ \\
\hline $25-29$ & $26 / 257(10.1)$ & $0.93(0.50$ to 1.71$)$ \\
\hline $30-34$ & $35 / 299(13.6)$ & 1.09 (0.62 to 1.93 ) \\
\hline $35-39$ & $42 / 213(19.7)$ & $2.02(1.16$ to 3.55$)$ \\
\hline $40+$ & $26 / 240(10.8)$ & 1 \\
\hline \multicolumn{3}{|l|}{ Ethnic group } \\
\hline White & $111 / 1063(10.4)$ & 1 \\
\hline Non-white & $21 / 140(15.0)$ & 1.51 (0.89 to 2.57$)$ \\
\hline \multicolumn{3}{|l|}{ Education after age of 16} \\
\hline None & $31 / 171(18.1)$ & 1 \\
\hline Up to 2 years & $30 / 203(14.8)$ & 0.78 (0.44 to 1.40$)$ \\
\hline 3 years or more & $67 / 725(9.2)$ & $0.46(0.28$ to 0.75$)$ \\
\hline Still in full time education & $3 / 98(3.1)$ & $0.14(0.03$ to 0.51$)$ \\
\hline \multicolumn{3}{|l|}{ Employment status } \\
\hline Employed & $99 / 1039$ (9.5) & 1 \\
\hline Unemployed & $32 / 166(19.3)$ & 2.26 (1.46 to 3.51$)$ \\
\hline \multirow{2}{*}{\multicolumn{3}{|c|}{$\begin{array}{l}\text { Aftended a HIV/GUM clinic in the past year } \\
\text { No }\end{array}$}} \\
\hline \multirow{3}{*}{$\begin{array}{l}\text { No } \\
\text { Yes }\end{array}$} & & \\
\hline & $37 / 680(5.4)$ & 1 \\
\hline & $93 / 515(18.1)$ & 3.83 (2.56 to 5.71$)$ \\
\hline \multicolumn{3}{|l|}{ Ever had an HIV test } \\
\hline Yes & $116 / 833$ (13.9) & 1 \\
\hline No & $15 / 269(4.1)$ & $0.26(0.14$ to 0.47$)$ \\
\hline
\end{tabular}

\section{Undiagnosed HIV infection}

Of the men whose saliva specimens tested HIV antibody positive, $54.5 \%(72 / 132)$ self reported their last HIV test result to be positive, $21.2 \%(28 / 132)$ reported their last HIV test to be negative, $12.1 \%$ (16/132) did not report their last HIV test result but perceived themselves to be HIV positive, $11.4 \%$ (15/ 132) either did not know their HIV status, perceived themselves to be HIV negative, or had not had an HIV test, and $0.8 \%(1 / 132)$ had missing data. Therefore, $32.5 \%(43 /$ 132) of HIV saliva antibody positive men were undiagnosed. The undiagnosed men were less likely currently to be unemployed $(\mathrm{OR}=0.15,95 \% \mathrm{CI} 0.04$ to 0.52$)$; however, there were no other demographic differences. Around half of both undiagnosed and diagnosed men reported UAI in the past year and over a quarter reported UAI with partners of an unknown or discordant HIV status (table 2). Of the HIV saliva antibody positive men who reported attending a GUM clinic in the past year $22.8 \%(21 / 92)$ were undiagnosed compared to $59.5 \%(22 / 37)$ of non-clinic attenders.

\section{HIV status by measures of sexual behaviour}

Men who reported having a sexually transmitted infection (STI) in the past year (table 3) were significantly more likely to be HIV saliva antibody positive than the men who had not had an STI in the past year (OR 2.16). There was a significant positive association between the number of UAI partners and HIV salivary antibody positive respondents. Men who had engaged in UAI with five or more partners in the past year were significantly more likely to be HIV saliva antibody positive than men who reported no UAI partners in the past year (OR 5.12). Also, those having one or more casual UAI partners in the past year were significantly more likely to be HIV saliva antibody positive than those having no casual UAI partners (OR 2.29). There was no association between HIV seropositivity and reported UAI partner concordancy. The associations remained significant when adjusting for age, education, and employment status.

\section{Reported and actual seroconcordant UAI partners}

Of the $83 \%$ (996/1206) of men in the sample who reported their current perceived HIV status, $4.1 \%$ (41/996) reported it incorrectly. Furthermore, of the 455 men who reported the numbers of concordant, discordant, and unknown UAI partners in the past year, 205 reported seroconcordant partners only; however, 75 of these men may have been mistaken in their assumption, as 10 men reported their last HIV status incorrectly, 51 men had never had an HIV test, and 14 reported not knowing their current HIV status.

Taking account of these incorrect assumptions, we estimate that only $29 \%$ of all men may have engaged in seroconcordant UAI in the past year, as opposed to $45 \%$ who reported that they had (fig 2 ).

\section{DISCUSSION}

We present 1 year's sexual behaviour data related to actual HIV status in a community sample of homosexual men. Over 1200 men were recruited from a variety of venues across London in 2000. Possible sources of bias have been described previously. ${ }^{12}{ }^{18}$ The $57 \%$ response rate to saliva testing and $74.4 \%$ overall response rate shows good acceptability of the survey. An internal consistency check was employed in the questionnaire to assess reliability and validity of self reported

Table 2 Reported high risk sexual behaviour among HIV saliva positive men

\begin{tabular}{llll}
\hline & Undiagnosed (\%) & Diagnosed (\%) & OR unadjusted (95\% CI) \\
\hline $\begin{array}{l}\text { \% who reported unprotected anal intercourse } \\
\text { with 1+ partners in the past year }\end{array}$ & $21 / 41(51.2)$ & $41 / 84(48.8)$ & 1.09 (0.52 to 2.29) \\
$\begin{array}{l}\text { \% who reported sexual partner with unknown } \\
\text { or discordant HIV status in past year }\end{array}$ & $12 / 38(31.6)$ & $22 / 84(26.2)$ & 1.20 (0.52 to 2.77) \\
\hline
\end{tabular}


Table 3 Measures of high risk behaviour (adjusting for age, education, and employment status using a logistic regression model)

\begin{tabular}{|c|c|c|c|}
\hline & \multirow{2}{*}{$\begin{array}{l}\text { HIV status } \\
\text { HIV positive/total (\%) }\end{array}$} & \multicolumn{2}{|c|}{ Odds ratio for positivity $(95 \% \mathrm{Cl})$} \\
\hline & & OR unadjusted & OR adjusted \\
\hline \multicolumn{4}{|l|}{ STI in the past year } \\
\hline No & $86 / 945(9.1)$ & 1 & 1 \\
\hline Yes & $41 / 231(17.7)$ & $2.16(1.41$ to 3.29$)$ & $2.13(1.40$ to 3.24$)$ \\
\hline \multicolumn{4}{|l|}{ UAI partners in the past year } \\
\hline 0 & $64 / 633(10.1)$ & 1 & 1 \\
\hline 1 & $26 / 304(8.6)$ & $0.83(0.50$ to 1.37$)$ & $0.92(0.56$ to 1.50$)$ \\
\hline 2 to 4 & 18/151 (11.9) & $1.20(0.66$ to 2.16$)$ & $1.27(0.72$ to 2.25$)$ \\
\hline $5+$ & $19 / 52(36.5)$ & $5.12(2.63$ to 9.93$)$ & $5.11(2.67$ to 9.76$)$ \\
\hline \multicolumn{4}{|l|}{ Casual UAI partners in the past year } \\
\hline 0 & $80 / 882(9.1)$ & 1 & 1 \\
\hline $1+$ & $46 / 247(18.6)$ & $2.29(1.52$ to 3.46$)$ & 2.21 (1.46 to 3.33$)$ \\
\hline \multicolumn{4}{|l|}{ Status of UAI partners } \\
\hline All partners of the same status & $26 / 205(12.7)$ & 1 & 1 \\
\hline 1+ partners discordant/unknown status & $34 / 250(13.6)$ & 1.08 (0.61 to 1.84$)$ & $1.05(0.60$ to 1.85$)$ \\
\hline
\end{tabular}

sexual behaviour. There is evidence of substantial consistency between questions (data not shown). Saliva testing has proved to be a valid method for detecting anti-HIV antibodies for surveillance purposes with good sensitivity and specificity $^{14} 19$ and has been tested previously in a community environment. ${ }^{20}$ Men who refused to take the saliva test were less likely to have attended a GUM clinic or have an HIV test in the past year. They were also more likely to report fewer UAI partners in the past year, suggesting that these men were at lower risk than those who accepted the saliva test which could lead to an overestimate of the HIV prevalence. A significant proportion of men reported an incorrect HIV status, and we cannot exclude the possibility that this could be due in part to questionnaire completion error. A potential error also exists among all reported GUM attendance in the past year as this question included HIV specific clinics, and a large number of HIV positive men were likely to have attended a HIV clinic in the past year.

Our data show that the prevalence of HIV in a sample of socially active homosexual men in London was high at $10.9 \%$. This is similar to data from homosexual GUM clinic attenders in London, among whom $11 \%$ tested HIV positive in the unlinked anonymous prevalence survey in 1999. In our sample $32.5 \%$ of HIV saliva antibody positive men remain undiagnosed. Data from the unlinked anonymous survey suggest that $25 \%$ of HIV positive homosexual men in the United Kingdom remain undiagnosed. ${ }^{21}$ Of the HIV saliva antibody positive clinic attenders in this survey $22.8 \%$ were undiagnosed, showing good consistency across the surveys. Despite emphasis on risk reduction counselling in clinics, men who were diagnosed were as likely to engage in high risk sexual behaviour as undiagnosed men.

There are high levels of risk behaviour and STIs in both HIV status groups, but this is seen particularly in the HIV saliva antibody positive men. A high proportion of HIV saliva antibody positive men continue to engage in high risk sexual behaviour after diagnosis, emphasising the need for focused health promotion programmes to reduce the risk of HIV transmission to others. Of those men engaging in any UAI, $45 \%$ reported same status only partners; there is, however, concern that $16 \%$ of men reporting same status only partners were either incorrect or unable to claim this with any certainty. They had either never had an HIV test, reported their status incorrectly, or did not know their current HIV status. HIV prevention strategies that encourage men to adopt negotiated safety as a risk reduction strategy have been promoted in the United Kingdom ${ }^{22}$ and abroad ${ }^{23} 24$ and it is therefore important for men to establish seroconcordancy through testing. A large proportion of men are also missing out on potential healthcare benefit, in particular highly active antiretroviral therapy. High risk men are more likely to attend a GUM clinic, suggesting that targeting clinics to increase HIV testing and to develop effective evidenced based health promotion interventions as suggested in the national health strategy, ${ }^{25}$ would be of great benefit.

Our data show an increase in reported UAI; similar trends have been reported by other community studies in the United Kingdom. ${ }^{26-28}$ For example, one community study reports, among men who had anal intercourse, UAI increased from $47 \%$ in 1998 to $56 \%$ in $2000 .{ }^{26}$ However there has not been a comparable increase in the incidence of HIV in this group. ${ }^{2}$ Men may be becoming more willing to report UAI or may be adopting various strategies which reduce the risk of HIV transmission. ${ }^{810}$ However our data suggest that there has been an increase in the proportion of men reporting UAI with partners of an unknown or discordant HIV status. A study conducted in London gyms also reports an increase in discordant or unknown UAI with casual partners from $14.5 \%$ in 1998 to $23.6 \%$ in $2001 .{ }^{28}$ Further qualitative and intervention research is required to understand the complexities of risk reduction strategies and to identify the particular health promotion or HIV prevention needs of HIV positive and negative men.

Trends data are essential for measuring the effectiveness of new health promotion initiatives focusing on these men and for monitoring the prevalence of HIV in a community sample of homosexual men.

\section{Key messages}

- There has been a significant increase since 1996 in the proportion of men reporting high risk sexual behaviour.

- HIV prevalence is high in this group of men with many infections remaining undiagnosed.

- HIV positive men were significantly more likely to report high risk sexual behaviour and STIs in the past year than the HIV negative men.

- Owing to the high levels of UAI and HIV prevalence in this group, the potential for onward transmission of HIV and increasing prevalence is a major public health concern. 
HIV prevalence is high in this group of homosexual men, but many infections remain undiagnosed. High levels of UAI continue to be reported by both HIV saliva antibody positive and negative men and the potential for onward transmission of HIV and increasing prevalence is a major public health concern.

\section{ACKNOWLEDGEMENTS}

We are grateful to Camden and Islington primary care trusts and the Department of Health for their financial support. We thank Camden and Islington Health Promotion who contacted all participating venues. We thank the Central Public Health Laboratory, in particular Sharon Barnett, for the testing of saliva samples.

\section{CONTRIBUTORS}

JPD, DEM, JVP, and AMJ initiated and designed the study; JPD managed the project; JVP coordinated the HIV testing; JPD analysed the data, wrote the first draft, and coordinated subsequent revisions of the paper; all authors contributed to the final version of this paper; JPD and DEM are the guarantors.

\section{Authors' affiliations}

J P Dodds, D E Mercey, A M Johnson, Centre for Sexual Health and HIV Research, Department of Primary Care and Population Sciences, Royal Free and University College Medical School, Mortimer Market Centre, Mortimer Market, off Capper Street, London WCIE 6AU, UK J V Parry, Sexually Transmitted and Bloodborne Virus Laboratory, Central Public Health Laboratory, London, UK

\section{REFERENCES}

1 PHLS AIDS Centre-HIV/STI Division. Communicable Disease Surveillance Centre and the Scottish Centre for Infection and Environmental Health. AIDS/ HIV Quarterly Surveillance Tables No 49:00/4.

2 Murphy G, Parry JV, Jordan LF, et al. STARHS test shows HIV transmission continuing in UK despite widespread HAART. 14th International AIDS Conference, Barcelona, Spain, 7-12 July 2002.

3 UK Collaborative Group on Monitoring the Transmission of HIV Drug Resistance, Analysis of prevalence of HIV-1 drug resistance in primary infections in the United Kingdom. BMJ 2001;322:1087-8.

4 PHLS (England, Wales \& Northern Ireland), DHSS and PS (Northern Ireland), Scottish ISD(D)5 Collaborative Group (ISD, SCIEH \& MSSVD). Trends in sexually transmitted infections in the United Kingdom: new episodes seen at genitourinary medicine clinics, 1995 to 2000. London: PHLS, 2001.

5 HIV and STI Division CDSC. HIV and AIDS in the UK-an epidemiological review 2000. London: PHLS, 2001.
6 Dodds JP, Nardone A, Mercey DE, et al. Increasing high risk sexual behaviour amongst gay men in London 1996-1998: a repeated cross sectional questionnaire study. BMJ 2000;320:1510-11.

7 Keogh P, Weatherburn P, Stephens M. Relative safety-risk and unprotected anal intercourse among gay men diagnosed with HIV. Sigma Research 1999.

8 Elford J, Bolding G, Maguire M, et al. Sexual risk behaviour among gay men in a relationship. AIDS 1999;13:1407-11

9 Van de Ven P, Kippax S, Crawford J, et al. In a minority of gay men, sexual risk practice indicates strategic positioning for perceived risk reduction rather than unbridled sex. AIDS Care 2002;4:471-80.

10 Kippax S, Crawford J, Davis M, et al. Sustaining safer sex: a longitudinal study of a sample of homosexual men. AIDS 1993;7:257-63.

11 Hunt AJ, Davies PM, McManus TJ, et al. HIV infection in a cohort of homosexual men and bisexual men. BMJ 1992;305:561-562.

12 Nardone A, Dodds JP, Mercey DM, et al. Active surveillance of sexual behaviour among homosexual men in London. Commun Dis Public Health 1998;1:197-201.

13 OraSure, Oral Specimen collection pad. Manufactured by Epitope, Inc, Beaverton, OR 97008, USA.

14 Parry JV, Perry KR, Mortimer P. Sensitive assays for viral antibodies in saliva; and alternative tests to serums. Lancet 1987;2:72-5.

15 Connell JA, Parry JV, Mortimer PP, et al. Novel assay for the detection of immunoglobulin $G$ anti-human immunodeficiency virus in untreated saliva and urine. J Med Virol 1993:41:159-64.

16 Epi-Info, Version 6.04b: a word processing, database and statistics program for public health. Atlanta: CDC, 1997

17 SPSS for Windows, Version 10.0.5 Chicago: SPSS Inc, 1999

18 Nardone A, Mercey DE, Johnson AM. Surveillance of sexual behaviour among homosexual men in a central London health authority. Genitourin Med 1997;73:198-202.

19 Johnson AM, Parry JV, Best SJ, et al. HIV surveillance by testing saliva. AIDS 1998;2:369-71.

20 Hunt AJ, Connell J, Christofinis G, et al. The testing of saliva samples for HIV-1 antibodies: reliability in a non-clinic setting. Genitourin Med 1993:69:29-30.

21 Department of Health. Prevalence of HIV and hepatitis infection in the United Kingdom 2000-annual report of the unlinked anonymous prevalence monitoring programme. London: DoH, 2001

22 Camden and Islington Community Health Services Trust. Thinking it through: a new approach to sex, relationships and HIV for gay men. London: Camden and Islington Community Services Trust, 1996.

23 Kippax S, Noble J, Prestage G, et al. Sexual negotiation in the AIDS era: negotiated safety revisited. AIDS 1997;11:191-7.

24 Ekstrand $M$, Stall R, Kegles $S$, et al. Safer sex among gay men: what is the ultimate goal. AIDS 1993;7:281-2.

25 Department of Health. The national strategy for sexual health and HIV London: DoH, 2001

26 Hickson F, Reid D, Weatherburn $P$, et al. Time for more: Findings from the National Gay Men's Sex Survey 2000. Sigma Research 2001.

27 Williamson LM, Hart GJ. Changes in HIV related sexual behaviour among gay men in Scotland, UK. Paper presented at 6th International AIDS Impact Conference, July 2003.

28 Elford J, Bolding G, Sherr L. High-risk sexual behaviour increases among London gay men between 1998 and 2001: what is the role of HIV optimism? AIDS 2002;16:1537-44. 